\title{
Dynamics and Control of a 3D Pendulum
}

\author{
Jinglai Shen, Amit K. Sanyal, Nalin A. Chaturvedi, \\ Dennis Bernstein, Harris McClamroch \\ Department of Aerospace Engineering \\ University of Michigan \\ Ann Arbor, MI 48109 \\ \{jinglais, asanyal, nalin, dsbaero, nhm\}@engin.umich.edu
}

\begin{abstract}
New pendulum models are introduced and studied. The pendulum consists of a rigid body, supported at a fixed pivot, with three rotational degrees of freedom. The pendulum is acted on by a gravitational force and control forces and moments. Several different pendulum models are developed to analyze properties of the uncontrolled pendulum. Symmetry assumptions are shown to lead to the planar 1D pendulum and to the spherical 2D pendulum models as special cases. The case where the rigid body is asymmetric and the center of mass is distinct from the pivot location leads to the 3D pendulum. Rigid pendulum and multi-body pendulum control problems are proposed. The 3D pendulum models provide a rich source of examples for nonlinear dynamics and control, some of which are similar to simpler pendulum models and some of which are completely new.
\end{abstract}

\section{INTRODUCTION}

Pendulum models have provided a rich source of examples in nonlinear dynamics and, in recent decades, in nonlinear control. A brief review is given of some of these models; this review provides appropriate background for the subsequent introduction of a new 3D pendulum model. The most common rigid pendulum model consists of a mass particle that is attached to one end of a mass-less, rigid link; the other end of the link is fixed to a pivot point that provides a rotational joint for the link and mass particle. If the link and mass particle are constrained to move within a fixed plane, the system is referred to as a planar 1D pendulum. If the link and mass particle are unconstrained, the system is referred to as a spherical 2D pendulum.

The published literature on such pendulum models is very large. Few publications view the pendulum as a rigid body. Standard pendulum models are defined by a single rotational degree of freedom, referred to as a planar rigid pendulum, or two rotational degrees of freedom, referred to as a spherical rigid pendulum. Control problems for planar and spherical pendulum models have been studied in [1], [2] and [3] and in many other references, such as [4], [5], [6], [7], [8], [9], [10], [11].

Numerous extensions of simple pendulum models have been proposed. These include various categories of elastic

This research has been supported in part by NSF under grant ECS0140053 . pendulum models and multi-body pendulum models. Interesting examples of multi-body pendulum models are: a pendulum on a cart, an acrobot, a pendubot, a pendulum actuated by a reaction wheel, the Furuta pendulum, and pendula consisting of multiple coupled bodies. Dynamics and control problems for these types of multi-body pendulum models have been studied in [10], [12], [13], [14], [15], [16], [17], [18].

Pendulum models are useful for both pedagogical and research reasons. They represent physical mechanisms that can be viewed as simplified academic versions of mechanical systems that arise in robotics and spacecraft. In addition to their important role in teaching the foundations of nonlinear dynamics and control, pendulum models have motivated the application of new research results in nonlinear dynamics and nonlinear control. This theme was recently emphasized in the plenary presentation given by Katsuhisa Furuta at the 2003 Conference on Decision and Control [2].

This paper introduces a new 3D pendulum model, analyzes its basic properties, and suggests several new associated control problems. The 3D pendulum is, simply, a rigid body, supported at a fixed pivot point, that has three rotational degrees of freedom; it is acted on by a uniform gravity force and, perhaps, by control and disturbance forces and moments.

This paper is a consequence of our continuing research on a laboratory facility, referred to as the Triaxial Attitude Control Testbed (TACT). The TACT has been constructed to provide a testbed for a variety of physical experiments on attitude dynamics and attitude control. The most important feature of the TACT design is that it is supported by a threedimensional air bearing that serves as an ideal frictionless pivot, allowing nearly unrestricted three degrees of rotation.

The TACT has been described in several prior conference publications [19], [20]. Issues of nonlinear dynamics for the TACT have been treated in [20], [21] and stability and control issues have been treated in [22], [23], [24], [25], [26]. The present paper is motivated by the realization that the TACT is, in fact, a physical implementation of a 3D pendulum. We are beginning to use the TACT 
to experimentally verify theoretical dynamics and control results that we obtained previously; we expect the TACT to provide a valuable experimental tool for continued research on advanced attitude dynamics and control problems.

\section{Mathematical Models for a Rigid Pendulum}

A rigid pendulum is a rigid body supported by a fixed, frictionless pivot, acted on by gravitational forces and disturbance and control forces or moments. A schematic of a rigid pendulum is shown in Figure 1.

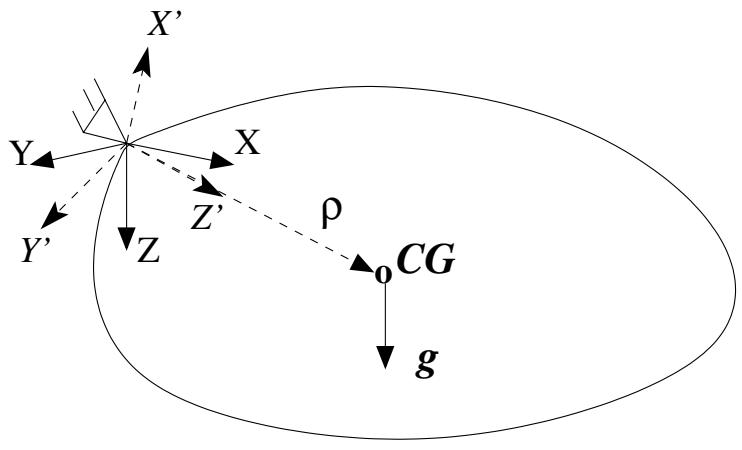

Fig. 1. A schematic of a Rigid Pendulum

The supporting pivot allows three degrees of rotational freedom of the pendulum. Uniform, constant gravity is assumed. The terminology 3D pendulum refers to the fact that the pendulum is a rigid body with three spatial dimensions and the pendulum has three rotational degrees of freedom.

Two coordinate frames are introduced. An inertial coordinate frame has its origin at the pivot; the first two coordinate axes lie in the horizontal plane and the third coordinate axis is vertical in the direction of gravity. A coordinate frame fixed to the pendulum body is also introduced. The origin of this body-fixed frame is also located at the pivot. In the body fixed frame, the moment of inertia of the pendulum is constant. This moment of inertia can be computed from the moment of inertia of a translated coordinate frame whose origin is located at the center of mass of the pendulum using the parallel axis theorem.

Rotation matrices can be used to describe the attitude of the rigid pendulum. A rotation matrix maps a representation of vectors expressed in the body-fixed coordinate frame to a representation expressed in the inertial coordinate frame. Rotation matrices provide global representations of the attitude of the pendulum, which is why they are utilized here. Other attitude representations, such as exponential coordinates, quaternions, or Euler angles, can also be used following standard descriptions. Hence the configuration of the rigid pendulum is a rotation matrix $R$ in $S O(3)$.
The associated angular velocity, expressed in the body-fixed coordinate frame, is denoted by $\omega$ in $\mathbb{R}^{3}$.

The equations of motion of a rigid pendulum are now presented. The constant inertia matrix, in the body-fixed coordinate frame, is denoted by the symbol $J$. The bodyfixed vector from the pivot to the center of mass of the pendulum is denoted by $\rho$. The symbol $g$ denotes the constant acceleration due to gravity. This is the data on which the equations of motion are based.

Standard arguments lead to the equations of motion for the rigid pendulum. In this section, it is assumed that there are no control or disturbance forces or moments. The dynamics are given by the Euler equation that includes the moment due to gravity:

$$
J \dot{\omega}=J \omega \times \omega+m g \rho \times R^{\mathrm{T}} e_{3} .
$$

The rotational kinematics equations are

$$
\dot{R}=R \widehat{\omega} .
$$

Equations (1) and (2) define the full dynamics of a rigid pendulum on the tangent bundle $T S O(3)$. In the above equations, $e_{3}$ denotes the third unit vector in the inertial coordinate frame, namely $e_{3}=(0,0,1)^{\mathrm{T}}$. The cross product notation $a \times b$ for vectors $a$ and $b$ in $\mathbb{R}^{3}$ is

$$
a \times b=\left[a_{2} b_{3}-a_{3} b_{2}, a_{3} b_{1}-a_{1} b_{3}, a_{1} b_{2}-a_{2} b_{1}\right]=\widehat{a} b,
$$

where, the skew-symmetric matrix $\widehat{a}$ is defined as

$$
\widehat{a}=\left[\begin{array}{ccc}
0 & -a_{3} & a_{2} \\
a_{3} & 0 & -a_{1} \\
-a_{2} & a_{1} & 0
\end{array}\right] .
$$

A special case occurs if the center of mass of the rigid pendulum is located at the pivot. In this case $\rho=0$, so that (1) given by Euler's equations with no gravity terms included. In the context of the rigid pendulum, this is referred to as the balanced case. Since there is a large literature on Euler's equations and the associated rotational kinematics, this case is not considered further in this paper. Rather, the focus of this paper is on the unbalanced case, where $\rho \neq 0$.

There are two conserved quantities for the rigid pendulum. First, the total energy, which is the sum of the rotational kinetic energy and the gravitational potential energy, is conserved. In addition, there is a rotational symmetry of the equations of motion corresponding to the group of rotations about the vertical line through the pivot. This symmetry leads to conservation of the component of angular momentum about the vertical axis through the pivot. These two results are summarized as follows.

Proposition 1: The total energy

$$
E=0.5 \omega^{T} J \omega-m g \rho^{T} R^{T} e_{3}
$$


and the component of the angular momentum vector about the vertical axis through the pivot

$$
h=\omega^{T} J R^{T} e_{3}
$$

are each constant along motions of the rigid pendulum.

The proof follows by showing that the time derivative of the total energy and the time derivative of the angular momentum component are each identically zero. This can be shown using the dynamics equation (1) and the kinematics equation (2).

\section{A. Reduced Equations of Motion}

The equations of motion (1) and (2) for a rigid pendulum are viewed as a model for the dynamics on the tangent bundle $T S O(3)$; these are referred to as the full equations of motion since they depend on the full attitude of the rigid pendulum. Since there is a rotational symmetry corresponding to the group of rotations about the vertical axis through the pivot and an associated conserved angular momentum component, it is possible to obtain a lower dimensional reduced model for the rigid pendulum. This reduction is based on the fact that the dynamics and kinematics equations can be written in terms of the reduced attitude vector $\Gamma=R^{\mathrm{T}} e_{3}$, which is the unit vector that expresses the gravity direction in the body-fixed coordinate frame.

Proposition 2: The dynamics of the $3 D$ pendulum given by (1) and (2) induce a flow on the tangent bundle $T S O(3) / S^{1}$ given by the reduced dynamics

$$
J \dot{\omega}=J \omega \times \omega+m g \rho \times \Gamma,
$$

and the reduced kinematics

$$
\dot{\Gamma}=\Gamma \times \omega .
$$

The proof follows from the definition of the reduced attitude vector $\Gamma$ and a demonstration that $\|\Gamma\|^{2}=1$.

Equations (5) and (6) are in a non-canonical form, but they are useful for studying the reduced dynamics. These equations can be easily modified to include control inputs, so long as the control inputs do not destroy the symmetry property that the angular momentum component about the vertical axis through the pivot is constant.

The reduced dynamics can also be expressed in other forms. In particular, the process of Routh reduction, as described in [27], leads to an alternative formulation of the reduced equations on $T S^{2}$. The equations are rather complex but they provide a different formulation making explicit that the reduced equations of motion are equivalent to two coupled rotational degrees of freedom.

\section{B. Special Cases of the Rigid Pendulum}

Two interesting special cases are now examined. It is assumed that the rigid pendulum is axisymmetric, that is two of the principal moments of inertia of the pendulum are identical and the pivot is located on the axis of symmetry of the pendulum. Assume that the body-fixed axes are selected so that $J=\operatorname{diag}\left(J_{t}, J_{t}, J_{a}\right)$ and $\rho=\rho_{s} e_{3}$ where $\rho_{s}$ is a positive scalar constant. Consequently, equation (5) can be written in scalar form, as

$$
\left\{\begin{array}{l}
J_{t} \dot{\omega}_{x}=\left(J_{t}-J_{a}\right) \omega_{y} \omega_{z}-m g \rho_{s} \Gamma_{y}, \\
J_{t} \dot{\omega}_{y}=\left(J_{a}-J_{t}\right) \omega_{z} \omega_{x}+m g \rho_{s} \Gamma_{x}, \\
J_{a} \dot{\omega}_{z}=0 .
\end{array}\right.
$$

From the last equation in (7), we see that the component of the pendulum angular velocity vector about its axis of symmetry is constant. This means that a constant $\omega_{z}$ defines an invariant manifold for the pendulum dynamics. The resulting reduced equations of motion, for constant $\omega_{z}$, are given by equations (6) and (7).

The special case that $\omega_{z}=0$ leads to an invariant dynamics of the axisymmetric rigid pendulum, described as follows:

Proposition 3: Assume the rigid pendulum has a single axis of symmetry and the pivot is located on the axis of symmetry of the pendulum as above. The equations of motion of the rigid pendulum define an induced flow on the tangent bundle $T S^{2}$ corresponding to $\omega_{z}=0$, given by the equations

$$
\begin{aligned}
J_{t} \dot{\omega}_{x} & =-m g \rho_{s} \Gamma_{y}, \\
J_{t} \dot{\omega}_{y} & =m g \rho_{s} \Gamma_{x}, \\
\dot{\Gamma} & =\Gamma \times \omega .
\end{aligned}
$$

These equations are said to represent a 2D spherical pendulum.

Now assume $\omega_{x}=0, \omega_{z}=0$ and $\Gamma$ is parameterized by an angle $\theta$ as $\Gamma=\left[\begin{array}{llll}-\sin \theta & 0 & \cos \theta\end{array}\right]^{\mathrm{T}}$. This leads to an invariant dynamics of the axisymmetric rigid pendulum, described as follows:

Proposition 4: Assume the rigid pendulum has a single axis of symmetry and the pivot is located on the axis of symmetry of the pendulum as above. The equations of motion of the rigid pendulum define an induced flow on the tangent bundle $T S^{1}$ corresponding to $\omega_{x}=0, \omega_{z}=0$ given by the equations

$$
\begin{aligned}
J_{t} \dot{\omega}_{y} & =-m g \rho_{s} \sin \theta \\
\dot{\theta} & =\omega_{y} .
\end{aligned}
$$

These equations are said to represent a 1D planar pendulum. 
Thus for an axially symmetric pendulum with the pivot located on the axis of symmetry, the well known 2D spherical pendulum and the 1D planar pendulum can be viewed as special cases of the pendulum dynamics described in Proposition 2. For an axially symmetric pendulum with the pivot located on the axis of symmetry, the induced dynamics corresponding to a nonzero value of $\omega_{z}$ is fundamentally different from the dynamics of the spherical pendulum; these dynamics seem not to have been previously studied. It should be emphasized that if the pendulum is asymmetric then the dynamics is general in the sense that neither the $2 \mathrm{D}$ spherical pendulum dynamics nor the 1D planar pendulum dynamics arise as special cases.

\section{The 3D Rigid Pendulum}

In the remainder of this paper, we assume the rigid pendulum is unbalanced, that is $\rho \neq 0$, and the rigid pendulum is asymmetric. These assumptions define the $3 \mathrm{D}$ rigid pendulum.

It should be emphasized that the full equations and the reduced equations for the $3 \mathrm{D}$ pendulum have been studied previously as models for heavy top dynamics [27], [28], [29], [30], [31]. However, the published literature, without exception, treats dynamics and control problems defined by relative equilibrium solutions rather than dynamics and control problems defined by usual equilibrium solutions that arise from the pendulum interpretation. This focus on the dynamics and control of equilibrium solutions of the full and reduced equations for the $3 \mathrm{D}$ pendulum is a novel perspective.

\section{EQUILIBRIA OF THE 3D PENDULUM}

To further understand the dynamics of the 3D pendulum, we study its equilibria. To simplify the equilibrium analysis it is convenient to choose the body-fixed frame so that its third axis is aligned with the vector from the pivot to the center of mass of the rigid pendulum. Thus, in the bodyfixed frame, $\rho=\rho_{s} e_{3}$, where $\rho_{s}>0$. Consequently, the gravity terms that appear in the dynamics equation (1) can be considerably simplified.

The equilibrium solutions for the 3D pendulum satisfy $\omega=0$ so that the attitude of the pendulum is constant. Substituting in equation (1), we obtain

$$
\rho \times R^{\mathrm{T}} e_{3}=0 .
$$

The above equation implies that the $3 \mathrm{D}$ pendulum is in equilibrium if and only if the center of mass vector $\rho$ is collinear with the gravity vector $R^{\mathrm{T}} e_{3}$ in the body-fixed coordinate frame. This implies that either the center of mass vector $\rho$ of the 3D pendulum points in the direction of the gravity vector or the center of mass vector $\rho$ points in the direction opposite to the gravity vector.
If $R_{e}$ defines an equilibrium attitude for the 3D pendulum, then a rotation of the $3 \mathrm{D}$ pendulum about the gravity vector by an arbitrary angle is also an equilibrium. Consequently, in $S O(3)$, there are two disjoint equilibrium manifolds of the 3D pendulum. The manifold corresponding to the first case in the above description is referred to as the hanging equilibrium manifold, since the center of mass is below the pivot for each attitude in the manifold. The manifold corresponding to the second case in the above description is referred to as the inverted equilibrium manifold, since the center of mass is above the pivot for each attitude in the manifold.

Next, we study the equilibria of the reduced equations (5) and (6). As before, the equilibria of the system satisfies $\omega=0$. Substituting in (5), we get

$$
\rho \times \Gamma=0 .
$$

Thus, we obtain two isolated equilibrium solutions of the reduced equations in $T S O(3) / S^{1}$ given by $\Gamma=e_{3}, \omega=0$, and by $\Gamma=-e_{3}, \omega=0$. The first corresponds to the hanging equilibrium where the center of mass is below the pivot and the second corresponds to the inverted equilibrium where the center of mass is above the pivot.

The stability of the two isolated equilibrium solutions of the reduced equations of motion is readily assessed. Presumably, the stability of the two families of equilibrium manifolds of the full equations of motion follows. The stability analysis can be based on the reduced equations.

Proposition 5: The hanging equilibrium $\Gamma_{h}=\frac{\rho}{\|\rho\|}$, of the reduced dynamics of the $3 D$ pendulum described by equations (5) and (6) is stable in the sense of Lyapunov. The hanging equilibrium manifold of the full dynamics of the $3 D$ pendulum described by equations (1) and (2) is stable in the sense of Lyapunov.

Proof: Consider the candidate Lyapunov function

$$
V(\omega, \Gamma)=\frac{1}{2} \omega^{\mathrm{T}} J \omega+m g\left(\|\rho\|-\rho^{\mathrm{T}} \Gamma\right)
$$

Note that $V\left(0, \Gamma_{h}\right)=0$ and $V(\omega, \Gamma)>0$ elsewhere. Furthermore, the derivative along a solution of (5) and (6) is given by

$$
\begin{aligned}
\dot{V}(\omega, \Gamma) & =\omega^{\mathrm{T}} J \dot{\omega}-m g \rho^{\mathrm{T}} \dot{\Gamma}, \\
& =\omega^{\mathrm{T}}(J \omega \times \omega+m g \rho \times \Gamma)-m g \rho^{\mathrm{T}}(\Gamma \times \omega), \\
& =\omega^{\mathrm{T}} m g \rho \times \Gamma-m g \rho^{\mathrm{T}} \Gamma \times \omega=0 .
\end{aligned}
$$

Thus, the hanging equilibrium is Lyapunov stable. The stability of the hanging equilibrium manifold of the full dynamics follows accordingly.

Proposition 6: The inverted equilibrium $\Gamma_{i}=-\frac{\rho}{\|\rho\|}$ of the reduced dynamics of the $3 D$ pendulum described by 
equations (5) and (6) is unstable. The inverted equilibrium manifold of the full dynamics of the $3 D$ pendulum described by equations (1) and (2) is unstable.

Proof: Consider the candidate Lyapunov function

$$
V(\omega, \Gamma)=\frac{1}{2} \omega^{\mathrm{T}} J \omega+m g\left(\|\rho\|+\rho^{\mathrm{T}} \Gamma\right) .
$$

Note that $V\left(0, \Gamma_{i}\right)=0$ and $V(\omega, \Gamma)>0$ elsewhere. Furthermore, the derivative of along a solution of (5) and (6) is given by

$$
\begin{aligned}
\dot{V}(\omega, \Gamma) & =\omega^{\mathrm{T}} J \dot{\omega}+m g \rho^{\mathrm{T}} \dot{\Gamma}, \\
& =\omega^{\mathrm{T}}(J \omega \times \omega+m g \rho \times \Gamma)+m g \rho^{\mathrm{T}}(\Gamma \times \omega), \\
& =\omega^{\mathrm{T}} m g \rho \times \Gamma+m g \rho^{\mathrm{T}} \Gamma \times \omega, \\
& =2 m g \omega^{\mathrm{T}}(\rho \times \Gamma),
\end{aligned}
$$

which is sign indefinite in a neighborhood of the inverted equilibrium. Thus, by Chetaev's theorem, the inverted equilibrium is unstable. The instability of the inverted equilibrium manifold of the full dynamics follows accordingly.

\section{CONTROL OF 3D RIGID PENDULUM}

The previous equations of motion for the $3 \mathrm{D}$ rigid pendulum can be modified to include control moments. In particular, equation (1) can be modified as

$$
J \dot{\omega}=J \omega \times \omega+m g \rho \times R^{T} e_{3}+M,
$$

where $M$ is the vector control moment on the $3 \mathrm{D}$ rigid pendulum, expressed in the body fixed coordinate frame. The specific form of the control moment depends on the control actuation assumptions.

One control actuation assumption, generalizing the development in [3], is that the pivot acceleration is the control input. In this case the control moment is

$$
M=m \rho \times R^{T} u,
$$

where $u$ denotes the pivot acceleration vector, expressed in the inertial coordinate frame. Such a control actuation assumption typically breaks the symmetry of the uncontrolled rigid pendulum dynamics. Consequently, the angular momentum component about the vertical axis through the pivot is not conserved and it is not possible to express the controlled dynamics in terms of the reduced attitude of the pendulum.

Another control actuation assumption, generalizing the developments in [21], [23], is that proof mass actuators are rigidly mounted on the rigid pendulum. Although the models in [21], [23] include actuation dynamics, it is possible to give a simple expression for the control moment as

$$
M=m g u \times R^{T} e_{3},
$$

if the dynamics of the proof mass actuators are ignored. Here the control input $u$ denotes the incremental displacement vector due to the proof mass motions expressed in terms of the body fixed coordinate frame so that the position vector of the center of mass of the $3 \mathrm{D}$ rigid pendulum is $\rho+u$ expressed in the body fixed coordinate frame. In this case, the mass $m$ denotes the total mass of the 3D pendulum and the proof mass actuators. It is easy to show that this type of control actuation does not invalidate conservation of the angular momentum component about the vertical axis through the pivot. It is clear that the resulting controlled dynamics can be expressed in terms of the reduced attitude vector.

It suffices here to mention that there are many stabilization and control problems for the $3 \mathrm{D}$ rigid pendulum that are analogous to problems for the 1D planar pendulum and for the 2D spherical pendulum.

Specific control problems for the $3 \mathrm{D}$ rigid pendulum based on the reduced dynamics on $T S O(3) / S^{1}$ include determining controllers that achieve one or more of the following objectives:

- Asymptotic stabilization of the hanging equilibrium of equations (5) and (6)

- Stabilization and asymptotic stabilization of the inverted equilibrium

- Swing up control, that is, making the inverted equilibrium of equations (5) and (6) an attractor

- Large domain of attraction of an equilibrium, that is, the domain of attraction of the hanging or the inverted equilibrium of equations (5) and (6)

Since feedback control can break the symmetry of rotations about the vertical axis through the pivot, the above problems can also be posed for the $3 \mathrm{D}$ rigid pendulum based on the full dynamics on $T S O(3)$. These problems include control of the complete attitude of the rigid body, including its rotation about the vertical axis through the pivot.

Each of the above control problems is considerably more challenging than the $1 \mathrm{D}$ planar pendulum version or the 2D spherical pendulum version of the problems. The challenges arise from the additional degrees of freedom and the nonlinear coupling between all of the degrees of freedom.

Additional control challenges arise if the 3D pendulum is underactuated, that is there are fewer than three independent control inputs. Interesting problems are the asymptotic stabilization of the $3 \mathrm{D}$ pendulum using two (or even one) independent control inputs. These underactuated 3D pendulum control problems have no analogies in the 1D planar pendulum or $2 \mathrm{D}$ spherical pendulum problems; these are new nonlinear control problems. 


\section{Control of A Multi-Body 3D Rigid PEndulum}

There is a large published literature on the dynamics and control of multi-body versions of 1D planar pendulums and 2D spherical pendulums. These include planar connections of two or more 1D planar pendulums using 1D planar joints, connections of two or more spherical pendulums using 2D spherical joints, a 1D planar pendulum connected to a cart that can translate, a 2D spherical pendulum connected to a cart that can translate, the Furuta pendulum, the 1D planar pendulum supporting a rotational wheel, and many other examples. Each one of these examples suggests extensions based on 3D pendula. The possible complexity of multibody $3 \mathrm{D}$ rigid pendulum systems is unlimited.

It is suitable to mention some of our prior work on the TACT since much of this work has been concerned with multi-body attitude systems, which are essentially multibody 3D pendulum systems. Modeling and formulation of control problems for multi-body 3D pendulum systems have been treated in [21]; in particular, models are developed for 3D pendulum systems that support mechanical systems such as reaction wheels (additional rotational degrees of freedom) or proof mass actuators (additional prismatic degrees of freedom). Stability results have been presented in [24], [25] and control results have been presented in [22], [23] for specific classes of multi-body attitude systems.

\section{CONCLUSIONS}

The main purpose of this paper is to motivate continuing research on dynamics and control problems for 3D rigid pendulums and for multi-body 3D rigid pendulum systems. Many of these problems have not been previously studied, and there are many opportunities for creative research.

\section{REFERENCES}

[1] A. S. Shiriaev, H. Ludvigsen, O. Egeland, "Swinging up the Spherical Pendulum via Stabilization of its First Integrals," Automatica, 40(1), January, 2004, 73-85.

[2] K. Furuta, "Control of Pendulum: From Super Mechano-System to Human Adaptive Mechatronics," Proceedings of $42^{\text {nd }}$ IEEE Conference on Decision and Control, December, 2003, 1498-1507.

[3] K.J. Astrom and K. Furuta, "Swinging-up a Pendulum by Energy Control," Proceedings of the IFAC Congress, Vol. E, 1996, 37-42.

[4] S. Mori, H. Nisihara and K. Furuta, "Control of Unstable Mechanical Systems: Control of Pendulum," International Journal of Control, 23, 1976, 673-692.

[5] C.C. Chung and J. Hauser, "Nonlinear Control of a Swinging Pendulum," Automatica, 31, 1995, 851-862.

[6] A.S. Shirieav, A. Pogromsky, H. Ludvigsen and O. Egeland, "On Global Properties of Passivity-based Control of an Inverted Pendulum," International Journal of Robust and Nonlinear Control, 10, 2000, 283-300.

[7] A.S. Shirieav, O. Egeland, H. Ludvigsen and A. Fradkov, "VSSversion of Energy-based Control of Swinging up of Pendulum," Systems \& Control Letters, 44, 2001, 45-56.

[8] A.S. Shirieav, O. Egeland, and H. Ludvigsen, "Global Stabilization of Unstable Equilibrium Point of Pendulum," Proceedings of the American Control Conference, 1999, 4034-4038.
[9] A.S. Shirieav, H. Ludvigsen, and O. Egeland, "Swinging up of the Spherical Pendulum," Proceedings of the IFAC World Congress, Vol. E, 1999, 65-70.

[10] A.S. Shirieav, H. Ludvigsen, O. Egeland, and A.L. Fradkov, "Swinging up of Simplified Furuta Pendulum," Proceedings of the European Control Conference, No. F1022-4, 1999.

[11] A.S. Shirieav, H. Ludvigsen, O. Egeland, and A. Pogromsky, "On the Global Properties of Passivity Based Control of the Inverted Pendulum," Proceedings of $38^{\text {th }}$ IEEE Conference on Decision and Control, 1999, 2513-2518.

[12] J.E. Marsden, J. Scheurle, and J.M. Wendlandt, "Visualization of Orbits and Pattern Evocation for the Double Spherical Pendulum," Proceedings of the ICIAM Conference, Hamburg, July, 1995.

[13] K. Furuta, T. Ochiai and N. Ono, "Attitude Control of a Triple Inverted Pendulum," International Journal of Control, 39, 1984, 13511365.

[14] T. Hoshino, H. Kawai and K. Furuta, "Stabilization of the Triple Spherical Inverted Pendulum-A Simultaneous Design Approach," Autommatisierungstechnik, 48, 2000, 577-587.

[15] J. Zhao and M.W. Spong, "Hybrid Control for Global Stabilization of the Cart-Pendulum System," Automatica, 37, 2001, 1845-1851.

[16] M.W. Spong, P. Corke, and R. Lozano, "Nonlinear Control of the Inertia Wheel Pendulum", Automatica, 37, 2001, 1845-1851.

[17] I. Fantoni, R. Lozano, and M.W. Spong, "Energy Based Control of the Pendubot," IEEE Transactions on Automatic Control, AC-45, 2000, 725-729.

[18] M.W. Spong, "The Swingup Control Problem for the Acrobot," IEEE Control Systems Magazine, 15, 1995, 72-79.

[19] D. S. Bernstein, N. H. McClamroch, and A. Bloch, "Development of Air Spindle and Triaxial Air Bearing Testbeds for Spacecraft Dynamics and Control Experiments," Proceedings of American Control Conference, Arlington, VA, June, 2001, 3967-3972.

[20] S. Cho, J. Shen, N. H. McClamroch, and D. S. Bernstein, "Equations of Motion of the Triaxial Attitude Control Testbed," Proceedings of 40th IEEE Conference on Decision and Control, Orlando, FL, December, 2001, 3429-3434.

[21] S. Cho, J. Shen and N. H. McClamroch, "Mathematical Models for the Triaxial Attitude Control Testbed," Mathematical and Computer Modeling of Dynamical Systems, Vol. 9, No. 2, 2003, 165-192.

[22] J. Shen, N. H. McClamroch and A. M. Bloch, "Local Equilibrium Controllability of the Triaxial Attitude Control Testbed," Proceedings of 41st Conference on Decision and Control, 2002, 528-533.

[23] S. Cho and N. H. McClamroch, "Feedback Control of Triaxial Attitude Control Testbed Actuated by Two Proof Mass Devices," Proceedings of 41st IEEE Conference on Decision and Control, 2002, 498-503.

[24] J. Shen, A. K. Sanyal, and N. H. McClamroch, "Asymptotic Stability of Multibody Attitude Systems," Stability and Control of Dynamical Systems with Applications: A Tribute to Anthony N. Michel, Birkhauser, 2003, 47-70.

[25] J. Shen, A. K. Sanyal, and N. H. McClamroch, "Asymptotic Stability of Rigid-Body Attitude Systems," Proceedings of 42nd Conference on Decision and Control, 2003, 544-549.

[26] A. Sanyal, M. Chellapa, J. L. Valk, J. Shen, J. Ahmed, and D. S. Bernstein, "Globally Convergent Adaptive Tracking of Spacecraft Angular Velocity with Inertia Identification," Proceedings of $42^{\text {nd }}$ Conference on Decision and Control, December 2003, 2704-2709.

[27] J.E. Marsden, T. Ratiu, and J. Scheurle, "Reduction Theory and the Lagrange-Routh Equations," Journal of Mathematical Physics, Vol 41(6), June 2000, 3379-3429.

[28] D. Lewis, T. Ratiu, J. C. Simo, and J. E. Marsden, "The Heavy Top: A Geometric Treatment," Nonlinearity, 5, 1992, 1-48.

[29] T.A. Posbergh and M.A. Egorov, "Robust stabilization of a Heavy Top," Proceedings of the $37^{\text {th }}$ IEEE Conference on Decision and Control, 1998, 3593-3598.

[30] C.J. Wan, V.T. Coppola, and D.S. Bernstein, "Global Asymptotic Stabilization of the Spinning Top," Optimal Control Applications \& Methods, 16, 1995, 189-215.

[31] K.Y. Lum, D.S. Bernstein, and V.T. Coppola, "Global Stabilization of the Spinning Top with Mass Imbalance," Dynamics and Stability of Systems, 10, 1995, 339-365. 